\title{
Optimization of electron microscopy for human brains with long-term fixation and fixed-frozen sections
}

Xiao-Bo Liu ${ }^{1}$ and Cynthia M Schumann $2,3^{*}$

\begin{abstract}
Background: Abnormal connectivity across brain regions underlies many neurological disorders including multiple sclerosis, schizophrenia and autism, possibly due to atypical axonal organization within white matter. Attempts at investigating axonal organization on post-mortem human brains have been hindered by the availability of high-quality, morphologically preserved tissue, particularly for neurodevelopmental disorders such as autism. Brains are generally stored in a fixative for long periods of time (often greater than 10 years) and in many cases, already frozen and sectioned on a microtome for histology and immunohistochemistry. Here we present a method to assess the quality and quantity of axons from long-term fixed and frozen-sectioned human brain samples to demonstrate their use for electron microscopy (EM) measures of axonal ultrastructure.

Results: Six samples were collected from white matter below the superior temporal cortex of three typically developing human brains and prepared for EM analyses. Five samples were stored in fixative for over 10 years, two of which were also flash frozen and sectioned on a freezing microtome, and one additional case was fixed for 3 years and sectioned on a freezing microtome. In all six samples, ultrastructural qualitative and quantitative analyses demonstrate that myelinated axons can be identified and counted on the EM images. Although axon density differed between brains, axonal ultrastructure and density was well preserved and did not differ within cases for fixed and frozen tissue. There was no significant difference between cases in axon myelin sheath thickness (g-ratio) or axon diameter; approximately $70 \%$ of axons were in the small $(0.25 \mu \mathrm{m})$ to medium $(0.75 \mu \mathrm{m})$ range. Axon diameter and g-ratio were positively correlated, indicating that larger axons may have thinner myelin sheaths.

Conclusion: The current study demonstrates that long term formalin fixed and frozen-sectioned human brain tissue can be used for ultrastructural analyses. Axon integrity is well preserved and can be quantified using the methods presented here. The ability to carry out EM on frozen sections allows for investigation of axonal organization in conjunction with other cellular and histological methods, such as immunohistochemistry and stereology, within the same brain and even within the same frozen cut section.
\end{abstract}

\section{Introduction}

White matter in the brain is comprised of axons that connect and convey information across regions. The majority of axons are myelinated and send signals over long distances [1,2]. Disruptions in the connectivity of these neural pathways may underlie many forms of psychiatric and neurodevelopmental disorders including multiple

\footnotetext{
* Correspondence: cschumann@ucdavis.edu

2University of California, Davis, School of Medicine, Department of Psychiatry and Behavioral Sciences, MIND Institute, Sacramento, USA

${ }^{3}$ UC Davis MIND Institute, 2805 20th Street, Sacramento, CA 95817, USA

Full list of author information is available at the end of the article
}

sclerosis, schizophrenia and autism [1,3-5]. One option for evaluating the integrity of neural pathways in the brain is to utilize magnetic resonance imaging (MRI) techniques such as diffusion tensor imaging (DTI). However, fine structural abnormalities associated with axons remain generally undetectable at the resolution of MRI and therefore require postmortem human brain tissue to investigate these uniquely human disorders at the cellular level with electron microscopy (EM).

A few recent studies have utilized postmortem human brain samples for EM analyses to detect abnormalities in axonal ultrastructure, including postmortem samples to

\section{Biomed Central}

(c) 2014 Liu and Schumann; licensee BioMed Central Ltd. This is an Open Access article distributed under the terms of the Creative Commons Attribution License (http://creativecommons.org/licenses/by/4.0), which permits unrestricted use, distribution, and reproduction in any medium, provided the original work is properly credited. The Creative Commons Public Domain Dedication waiver (http://creativecommons.org/publicdomain/zero/1.0/) applies to the data made available in this article, unless otherwise stated. 
evaluate white matter in autism [5], schizophrenia [6] and surgically-removed tissue from epilepsy patients [7]. However, the often small sample sizes and limited human brains available for studies of axonal organization significantly impede progress in our understanding of these neurological and psychiatric disorders. In reality, many human brains have been fixed in formalin for long periods of time (over 10 years) in suboptimal conditions, and/or frozen and sectioned for neuropathology or immunohistochemistry. Therefore assessing the fine structure of axons with EM can often be problematic or preclude quantitative analyses. In addition, brain samples for the study of pediatric and neurodevelopmental disorders are rare and must be shared amongst several investigators employing different approaches and methods.

New approaches to preserve ultrastructural quality of human brain white matter and evaluate long term fixed and frozen samples are highly necessary in order to obtain quantitative information about the structural integrity of axon pathways in diseased and normal human brains. The benefits of developing such protocols are two-fold: One, it would increase the quantity of available tissue to share by utilizing preexisting long-term fixed and/or fixed-frozen sectioned brains, and two, the ability to apply other analyses, such as immunohistochemistry and stereology, to adjacent EM sections in order to evaluate multiple neuropathological mechanisms within the same brain. Here we present a modified method [8] from non-human primate tissue studies for optimal preservation and quantitative EM analyses using human brain tissues with long term fixation and flash-frozen cut human brain sections. The protocols presented here will allow for many more high quality human brains to be available and usable for study of ultrastructural changes in the white matter of the human brain.

\section{Materials and methods Tissue procurement}

Three human brains were acquired in 2001 from the Department of Pathology at the University of California, Davis School of Medicine. All three cases were typically developing males with no major neurological or psychiatric conditions affecting the brain; the cause of death in each case was myocardial infarction. Case 1: 61 year old, postmortem interval (PMI, from time of death to fixation) 36 hours. Case 2: 66 year old, PMI 16 hours. Case 3: 44 year old, PMI 26 hours. After removal of the brain from the skull, each brain was bisected into two hemispheres. The right hemisphere was immersed in 10\% buffered formalin and stored at $4^{\circ}$ celsius in our laboratory for three years. The formalin in each container was replaced with fresh $10 \%$ buffered formalin approximately every two years. After three years, the right hemisphere from each case was coronally sectioned into $\sim 5 \mathrm{~cm}$ thick blocks and processed as follows (Table 1).

\section{Case 1 and 2}

The tissue block containing the superior temporal cortex from each case at the level of the mid-rostocaudal hippocampus (Figure 1) was isolated and stored in 10\% buffered formalin for an additional nine years at $4^{\circ} \mathrm{C}$. An approximately $1 \mathrm{~cm}^{3}$ tissue block was then dissected from each to include the white matter dorsal (below) to the superior temporal cortex (Figure 1). Case 1 was further divided into two samples containing the medial (Case 1a) and lateral (Case $1 \mathrm{~b}$ ) portions to assess tissue quality within a single brain. Three tissue blocks (Case 1a, 1b, and Case 2) were photographed and transferred to $2 \%$ paraformaldehyde plus $2.5 \%$ glutaraldehyde in $0.1 \mathrm{M}$ phosphate buffer solution $\left(4^{\circ} \mathrm{C}\right)$; then fixed for at least two weeks. After three rinses in cold $0.1 \mathrm{M}$ phosphate buffer, the blocks were further trimmed to approximately $1 \mathrm{~cm} \times 1 \mathrm{~cm} \times 0.5 \mathrm{~cm}$ in size. The blocks were cut with a vibratome (Leica) at 70-80 $\mu \mathrm{m}$ thickness; sections were collected and stored in cold 0.1M phosphate buffer $(\mathrm{PB})$ in preparation for EM processing. An additional $1 \mathrm{~cm}^{3}$ tissue block was sampled from each case (1 and 2) and placed into a cryoprotectant solution (10\% glycerol for two days and $20 \%$ glycerol) for five days in preparation for freezing. Each tissue block was flash frozen with 2-methyl butane (isopentane) and serially sectioned coronally into $50 \mu \mathrm{m}$ thick sections. Two sections from each case were then washed in $\mathrm{PB}$ and transferred into $2 \%$ paraformaldehyde and $2.5 \%$ glutaraldehyde in $0.1 \mathrm{M}$ phosphate buffer solution $\left(4^{\circ} \mathrm{C}\right)$ for 14 hours, then stored in cold $0.1 \mathrm{M}$ phosphate buffer in preparation for EM processing.

\section{Case 3}

A $2 \mathrm{~cm} \times 3 \mathrm{~cm} \times 6 \mathrm{~cm}$ temporal lobe block from Case 3 , after three years of storage in $10 \%$ buffered formalin at $4^{\circ} \mathrm{C}$, was placed into a cryoprotectant solution ( $10 \%$ glycerol for two days and $20 \%$ glycerol) for five days in preparation for freezing. The tissue block was flash frozen with 2-methyl butane (isopentane) and serially sectioned coronally into six series of $50 \mu \mathrm{m}$ thick sections and two series of $100 \mu \mathrm{m}$ thick sections. One $100 \mu \mathrm{m}$ series was stained with $0.25 \%$ thionin (standard Nissl method) for previously published stereological analyses [9]. One series of $50 \mu \mathrm{m}$ thick sections was stored in tissue cryoprotectant solution (TCS) at $-80^{\circ} \mathrm{C}$ for an additional nine years. From this series, three $50 \mu \mathrm{m}$ frozen cut sections through the mid-rostrocaudal hippocampus were selected and washed in PB and transferred into $2 \%$ paraformaldehyde and $2.5 \%$ glutaraldehyde in $0.1 \mathrm{M}$ phosphate buffer solution $\left(4^{\circ} \mathrm{C}\right)$ for 14 hours, then stored in cold $0.1 \mathrm{M}$ phosphate buffer in preparation for EM processing.

\section{Electron microscopic processing}

Basic steps for EM processing are described in Table 1. For each case, approximately 4-5 sections were washed 
Table 1 Tissue preparation and processing methods for electron microscopic imaging

\begin{tabular}{lll}
\hline Steps & Long-term formalin fixed human brains & Fixed-frozen sectioned human brains \\
\hline 1 & Postfixed with $4 \%$ paraformaldehyde $+2.5 \%$ glutaraldehyde in $0.1 \mathrm{M} \mathrm{PB}$ for 2 weeks at $4^{\circ} \mathrm{C}$ & $\begin{array}{l}\text { Postfixed with } 4 \% \text { paraformaldehyde }+2.5 \% \\
\text { glutaraldehyde in } 0.1 \mathrm{M} \mathrm{PB} \text { for } 14 \text { hours at } 4^{\circ} \mathrm{C}\end{array}$ \\
2 & Vibratome section at $80 \mu \mathrm{m}$, store in $0.1 \mathrm{M} \mathrm{PB}$ & Washed and stored in $0.1 \mathrm{M} \mathrm{PB}$ \\
3 & Osmification in $\mathrm{OsO}_{4} 0.1 \mathrm{M} \mathrm{PB}$ for 20 min and washed in $0.1 \mathrm{M} \mathrm{PB}$ & \\
4 & Dehydration in graded ethanol solutions and $100 \%$ acetone \\
6 & $1: 1$ acetone/Araldite for 1 hour at room temperature and in pure Araldite overnight at $4^{\circ} \mathrm{C}$ \\
7 & Flat embedding on siliconized slides with Araldite \\
8 & Polymeriztion at $60^{\circ} \mathrm{C}$ for 48 hours in oven \\
9 & Dissection of white matter from embedded sections and glue to blank resin blocks & \\
10 & Thin sectioning at $70-80 \mathrm{~nm}$ and stain grids with uranyl acetate and lead citrate solutions & \\
\hline
\end{tabular}

extensively in $0.1 \mathrm{M}$ phosphate buffer, transferred to $2 \%$ $\mathrm{OsO}_{4}$ in $0.1 \mathrm{M} \mathrm{PB}$ for $20 \mathrm{~min}$, and then washed three times in cold $0.1 \mathrm{M}$ phosphate buffer. Sections were dehydrated in $70 \%, 90 \%, 95 \%$ and $100 \%$ ethanol and finally in $100 \%$ acetone for fifteen minutes. Sections were incubated in 1:1 Acetone/Araldite solution for one hour at room temperature, and transferred to pure Araldite for storage overnight at $4^{\circ} \mathrm{C}$. Sections were embedded between silicon (Sigmacote, Sigma) coated coverslips and glass slides in freshly prepared Araldite and polymerized in $60-70^{\circ} \mathrm{C}$ oven for 48 hours. After polymerization, embedded sections were examined under a light microscope. The core of white matter approximately 2-3 mm below the gray-white matter boundary dorsal to the superior temporal gyrus, as shown in Figure 1, was identified and cut under a dissecting microscope; each piece of tissue was glued to a blank resin block. Each section was further trimmed and oriented on an ultramicrotome (Leica). We confirmed that the section contained the desired white matter by examining semi-thin sections $(0.5 \mu \mathrm{m})$ stained by toluidine blue. Ultrathin sections at 70-80 $\mathrm{nm}$ were cut on the ultramicrotome

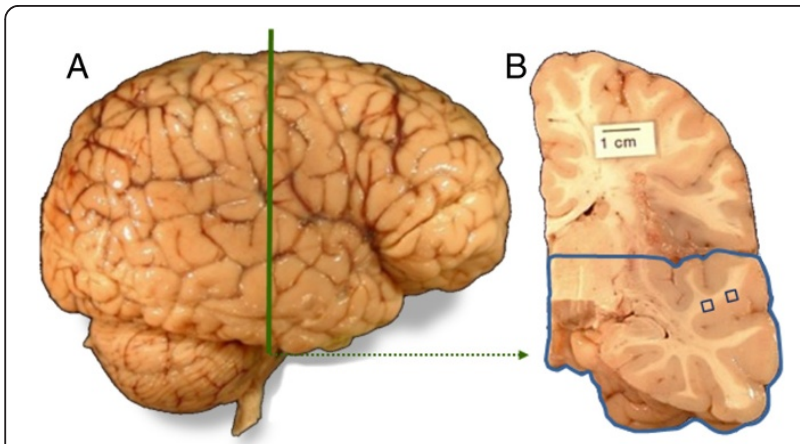

Figure 1 Location of white matter sampling sites under STG. (A) Lateral view of human brain showing the STG. Vertical line indicates location of coronal cross-section in (B). (B) Coronal brain slab through mid-rostrocaudal STG; squares indicate location of samples prepared for EM and collected on Formvar coated single slot grids (Electron Microscopy Sciences). Grids were stained with uranyl acetate and lead citrate solutions, dried and stored in a grid box for EM imaging.

\section{Electron microscopic imaging}

Grids were examined on a Philips CM120 Electron Microscope at $80 \mathrm{kV}$. Low magnification images of white matter regions containing axon bundles were taken at $\mathrm{X} 4,800$ or X7, 000. To visualize the details of axons and other subcellular elements, additional images were taken at $\mathrm{X} 15,000$ or X20,000, so that the myelin sheaths and subcellular organelles such as mitochondria and neurofilaments could be clearly identified. From the EM samples examined, axons with myelin sheaths and axoplasmic organelles fit ultrastructural criteria for identification [10]. EM images were captured using a high resolution $2 \mathrm{~K}$ X $2 \mathrm{~K}$ CCD camera (Gatan, Pleasanton, CA) and imported into DigitalMicrograph (Gatan). To prepare Figures 2, 3, and 4, images were processed and imported to Adobe Photoshop (Adobe Systems, Mountain View) for further adjustments of brightness and contrast.

\section{Electron microscopic analysis and quantification}

EM images were converted and saved as Tiff format to obtain quantitative measurements in Image J (NIH). We then carried out three types of analyses: (1) axon number density, (2) axon size, and (3) myelin sheath thickness (g-ratio), as described below.

\section{Axon density}

This method was based on counting the number of myelinated axons in the defined unit area (imaging field of $560 \mu \mathrm{m}^{2}$ at X7260), thus, the density of axons can be calculated as the number of axons per square micron.

\section{Axon size}

Images were imported to Image $J$ and the diameter of each axon was measured. Case $1(\mathrm{a}, \mathrm{b})$ was pooled 


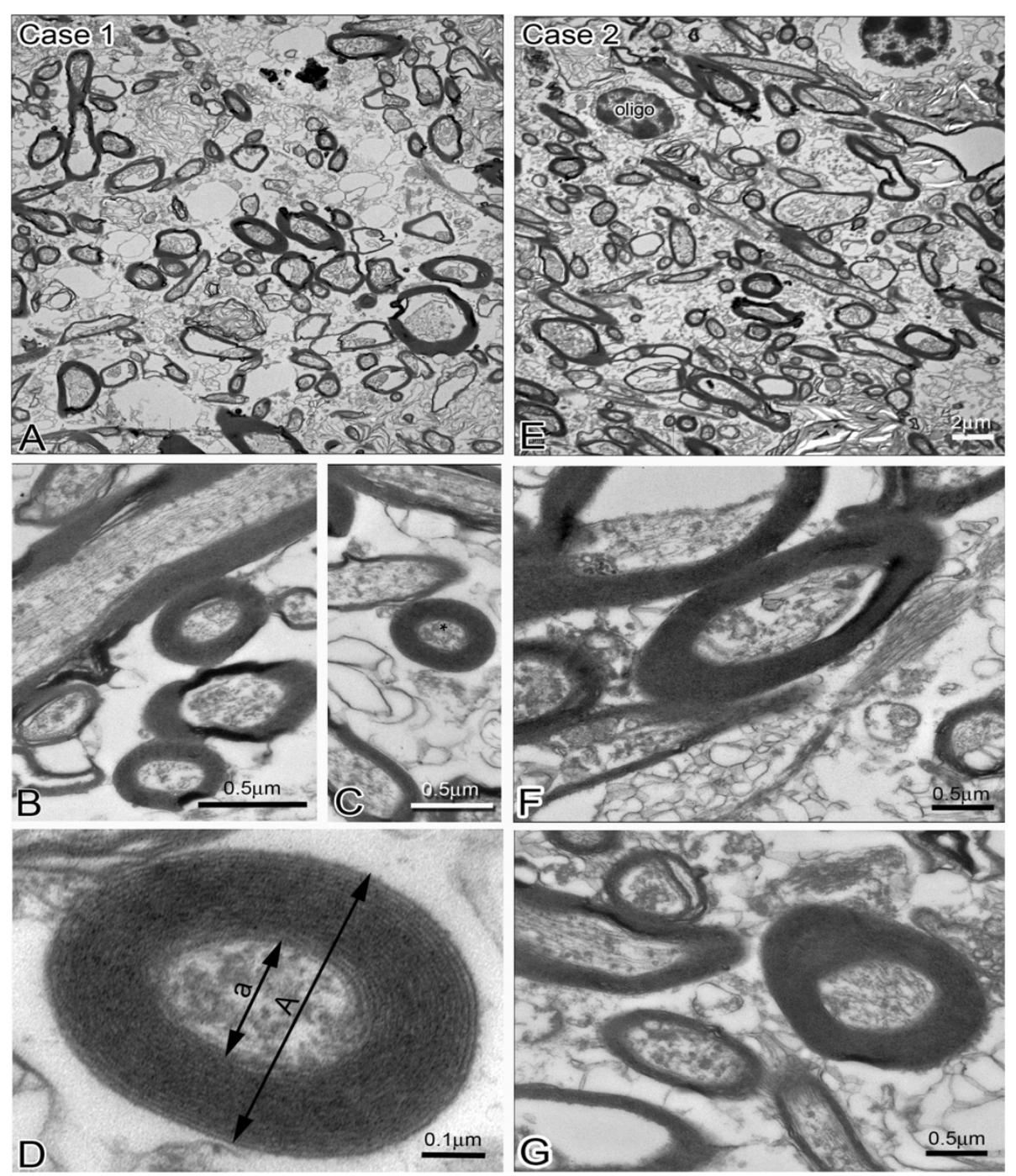

Figure 2 Electron micrographs of myelinated axons in white matter below STG taken from two human brain samples case 1 and case 2. (A): Low magnification electron micrograph shows numerous myelinated axons of varying sizes in case 1. (B, C) High magnification EM demonstrates ultrastructural details of myelinated axons in cross and sagittal orientation. Note fine structure of microtubules and other subcellular organelles can be identified. The compactness of myelin sheaths differs among axons; bars $=0.5 \mu \mathrm{m}$. (D) Higher magnification EM of thick compact myelinated axon indicated in (C) by asterisk. Layers of myelin sheath can be clearly identified and counted. Axon diameter is defined by double arrow pointing to axoplasmic membranes labeled as $\mathbf{a}$, the diameter of the myelinated fiber is defined by a double arrow pointed to the out layers of myelin sheath labeled as $\mathbf{A}$; $\mathbf{g}$-ratio $=\mathrm{a} / \mathrm{A}$. bar $=0.1 \mu \mathrm{m}$. (E) Low magnification EM shows myelinated axons and a few oligodendrocytes cell bodies in case 2. Note space between axon profiles is smaller compared to $(\mathbf{A})$ indicating better structural preservation; bar $=2 \mu \mathrm{m}$. $(\mathbf{F}, \mathbf{G})$ High magnification EM showing some myelinated axons of varying sizes and myelin thickness. Note microtubules and neurofilaments can be clearly identified within axons; bar $=0.5 \mu \mathrm{m}$.

together for axon size analyses. We divided axons into groups based on diameter measures: small $0.25-0.5 \mu \mathrm{m}$; medium $0.5-0.8 \mu \mathrm{m}$; large $>0.8 \mu \mathrm{m}$.

\section{Myelin sheath thickness}

g-ratio calculation provides information about the thickness of the myelin sheath of individual axon fibers [11,12]. A high magnification image $(\mathrm{X} 21,000)$ of myelinated axons was obtained and the image was imported to Image J for measuring the diameter of the axon (a, Figure 2D) and the myelinated axon caliber (A, Figure 2D). We measured at least 30 myelinated axons randomly chosen from each case. The ratio was calculated as the diameter of the axon (a) divided by diameter of the myelinated axon caliber (A): g-ratio = $\mathrm{a} / \mathrm{A}$. Thus, the smaller the g ratio, the thicker the myelin sheath layer.

This study was exempt from Internal Review Board approval at UC Davis. 


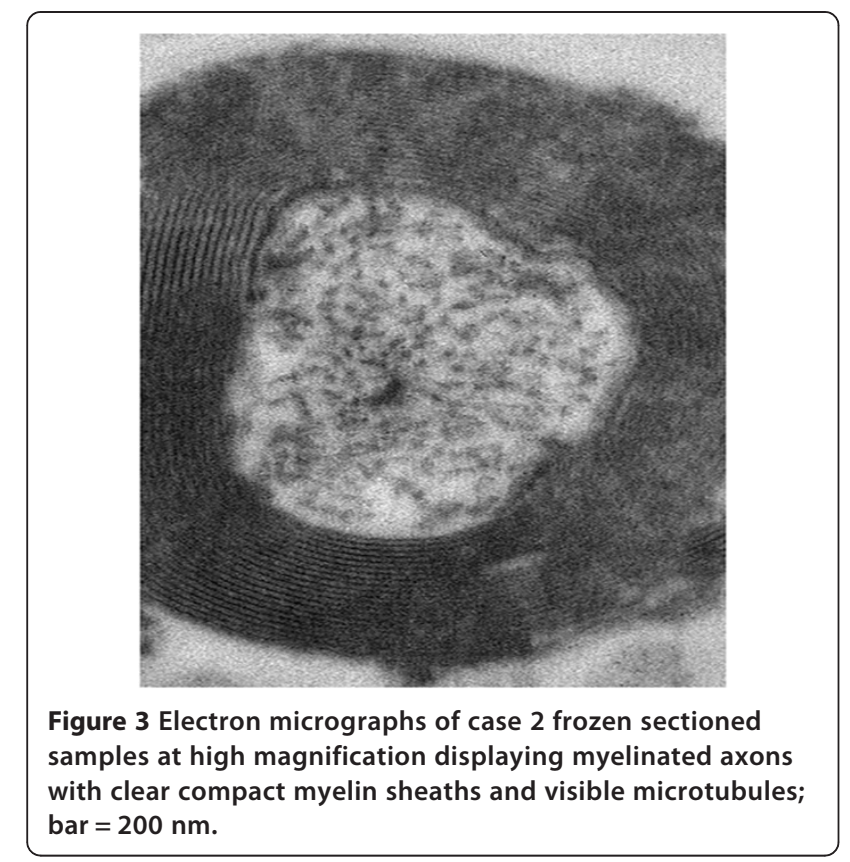

\section{Results}

Qualitative observations of ultrastructural features in white matter

EM images (Figure 2A and E) at low magnification demonstrate the distribution pattern of myelinated axons in white matter of the superior temporal gyrus from fixed (never frozen) tissue. Surprisingly, axonal ultrastructure was well preserved and remarkably consistent between the fixed and fixed-frozen tissue samples collected from each of the cases 1 and 2. Compared to case 1, case 2 though exhibited considerably denser organization of myelinated axons (myelinated axons $/ \mu \mathrm{m}^{2}$ ). The extraaxonal space was larger in case 1 samples than case 2, which also contained more identifiable structural contents (Figure 2F and 2G). Case 2 sample also preserved some cellular components, including oligodendrocyte cell bodies (Figure 2E). To visualize the structural details of myelin sheaths and quantify the myelin thickness, some myelinated axons were examined at higher magnification $(\times 21,000)$ (Figures 2D, 3 and $4 \mathrm{D})$. In these images, myelinated axons from case 1

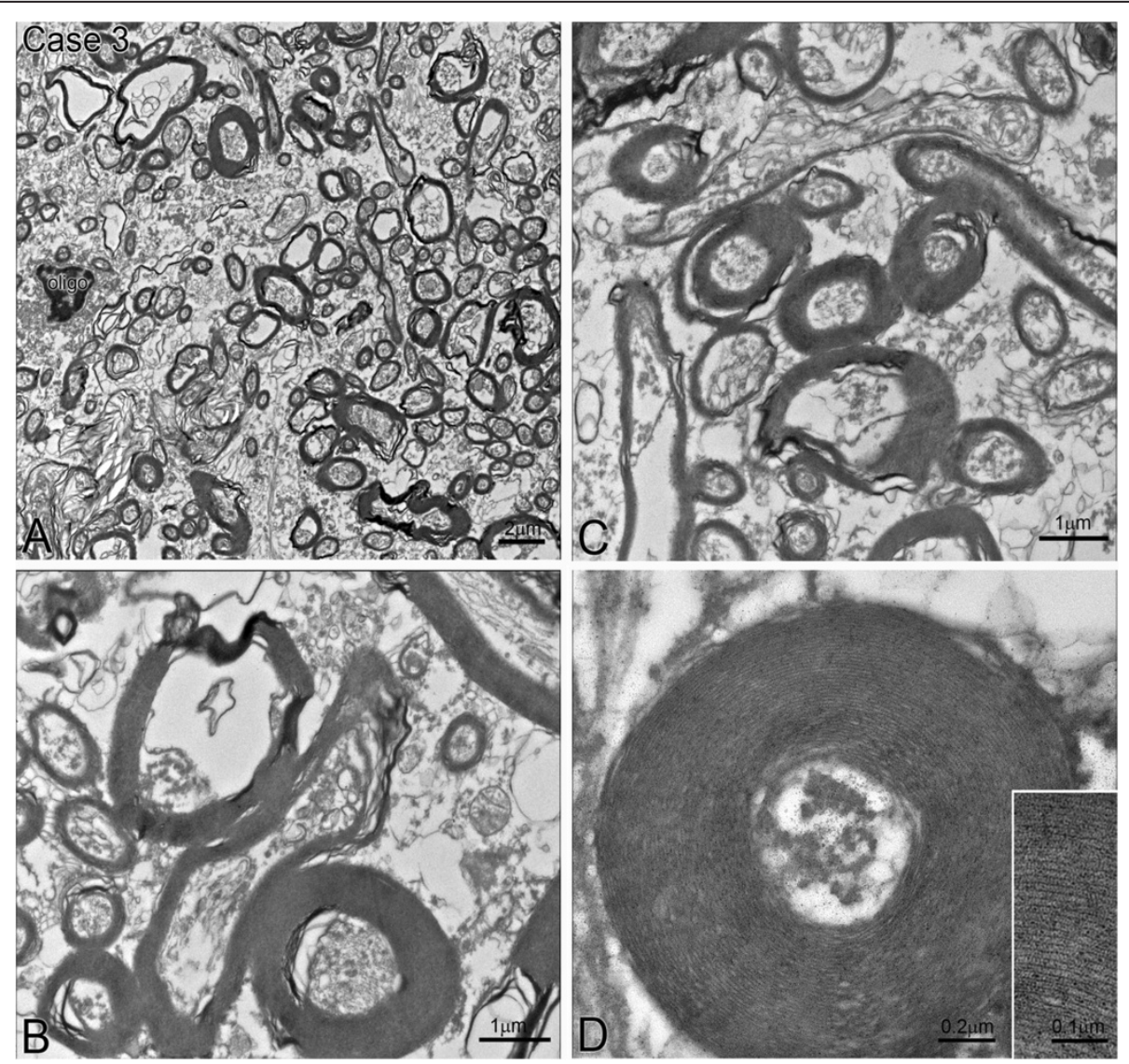

Figure 4 Electron micrographs showing high white matter ultrastructural quality in frozen cut sections from case 3. (A) LoW magnification EM displaying numerous myelinated axons of varying sizes. Note an oligodendrocyte cell body located on left side of images; bar $=2 \mu \mathrm{m}$. (B, C) High magnification EM images showing myelinated axons with some subcellular organelles preserved, such as microtubules, neurofilaments and a few mitochondria; bars $=1 \mu \mathrm{m}$. (D) Higher magnification EM image showing a myelinated axon with numerous compact myelin sheaths. The inset demonstrates clarity of myelin sheath layers; bar $=0.2 \mu \mathrm{m}$, bar in inset $=0.1 \mu \mathrm{m}$. 
and 2 samples displayed similar ultrastructural features (Figure 2B, C, F and G), the myelin sheaths were in general very well preserved. The appearances of axoplasmic contents were variable, but in many images, microtubules and neurofilaments are clearly identifiable, whereas mitochondria were less easily identifiable. Axon ultrastructure appeared to be well preserved with numerous compact myelin sheaths, regardless of freezing, as showed in Figure 3 for case 2.

In case 3, fixed-frozen sectioned samples contained well-defined oligodendrocytes among myelinated axons, some subcellular organelles such as microtubules and neurofilaments, as well as mitochondria, could also be identified (Figure 4A-4C). At higher magnification, the layers of myelin sheath could be identified and counted. The axon diameter (a) and myelinated fiber diameter (A) were clearly identifiable and therefore could be quantified as described below.

\section{Quantitative analysis and comparison of different brain samples}

Density of myelinated axons in the white matter

We counted the total number of myelinated axons in each EM image field $\left(560 \mu \mathrm{m}^{2}, \times 7260\right)$ to calculate mean myelinated axon density (per square micron region) for each sample (Figure 5A). There was a significant difference across all 6 samples in mean axon density (ANOVA, $\mathrm{p}<.001$ ). As noted in our qualitative observations, we confirmed that all case 1 samples $\left(\right.$ mean $=0.14 \pm 0.01$ axons $/ \mu \mathrm{m}^{2}$ ) are significantly less $(\mathrm{p}<0.05)$, irrespective of freezing, than both case 2 and case 3 samples, which have densities $0.17 \pm 0.02$ axons $/ \mu \mathrm{m}^{2}$ (case 2 ) and $0.19 \pm 0.02$ axons $/ \mu \mathrm{m}^{2}$ (case 3 ) respectively. There is no significant difference ( $\mathrm{p}=0.12$ ) between case 2 and 3 samples. Within case 1 , there was no difference in axon density between the two fixed samples (1a and $1 \mathrm{~b}$ ) and the frozen sample (ANOVA, $\mathrm{p}=.94$ ). There was also no difference in axon density (mean $=0.17 \pm 0.02$ axons $/ \mu \mathrm{m}^{2}$ ) between the fixed and frozen samples from case 2 (ANOVA, $\mathrm{p}=.98$ ). These findings indicate that, although axon density differs between typically-developing brains, axonal ultrastructure is preserved through the freezing process presented here and does not impact measures of myelinated axon density.

\section{Size distribution of myelinated axons in the white matter}

Axon diameter was measured in approximately 300 myelinated axons from each case. As shown in Figure 5B, we confirmed that the size distribution patterns of case 1 , case 2 and case 3 are nearly identical irrespective of freezing preparation. Interestingly, in these samples, more than $70 \%$ of the axon diameters are in the range of $0.25 \mu \mathrm{m}$ to $0.75 \mu \mathrm{m}$, indicating the majority of axons in the white matter under superior temporal cortex are in the small $0.25-0.5 \mu \mathrm{m}$ to medium 0.5-0.8 $\mu \mathrm{m}$ size range. Mean axon diameters are as follows: Case1: $0.80 \mu \mathrm{m}$; case 2: $0.85 \mu \mathrm{m}$; case 3: $0.84 \mu \mathrm{m}$.

\section{g-ratio analysis of myelin sheath thickness}

As shown in Figure 5C, mean g-ratio for each sample is as follows: case 1a: $0.64 \pm 0.10$; case $1 \mathrm{~b}$ : $0.66 \pm 0.11$; case 2: $0.64 \pm 0.09$ and case 3: $0.61 \pm 0.12$. No significant difference was found in comparing all four samples (ANOVA, $\mathrm{p}=0.7$ ). There was a moderate difference between case 2 and $3(\mathrm{p}<0.07)$. We then carried out a population analysis by examining g-ratio distributions and the correlation with axon diameters. As shown in Figure 5D, the g-ratio for each sample overlaps extensively for small to medium sized axons $(0.25$ to $0.75 \mu \mathrm{m}$ in diameter); this result is consistent with the axon size distribution (Figure 5B). Interestingly, there is a positive correlation between g-ratio number and axon diameter $(\mathrm{r}=.38, \mathrm{p}<.01)$, indicating that larger axons may have slightly thinner myelin sheaths.

\section{Discussion}

In the present study, we sought to optimize methods for utilizing EM to examine the ultrastructural quality of white matter samples from long-term formalin fixed human brain tissue blocks and flash-frozen cut human brain sections. Using a modified EM protocol from previous animal studies [8,13-17], we analyzed three longterm fixed human brain samples and three fixed-frozen sectioned human brain sample. For the first time, this study demonstrates that white matter ultrastructure is well preserved and can be quantified in formalin fixedfrozen human brain sections, in addition to long term fixed human tissue sections, for studies of human brain neuropathology.

Qualitatively, we found that fixed-frozen sectioned tissue does maintain axonal ultrastructure in addition to well-defined oligodendrocytes, subcellular organelles, and clearly delineated layers of myelin sheath with the protocol presented here. There was no clear difference in axonal ultrastructure between tissue sections that had previously been frozen and those that had not. However, one of the two long term fixed brains (case 1: 61 year old, PMI 36 hours) displays an apparent decrease in myelinated axon density relative to the other long term fixed brain (case 2: 66 year old, PMI 16 hours) and to the fixed-frozen sectioned brain (case 3: 44 year old, PMI 26 hours), indicating that factors other than freezing may alter axon density, such as agonal state after death, PMI, variable storage time, or unknown brain pathology.

Quantitatively, confirming our qualitative observations, case 1 has significantly lower myelinated axon density relative to cases 2 and 3, irrespective of freezing. There is no significant difference in axon size distribution in all three brains, with diameters in the small to medium 


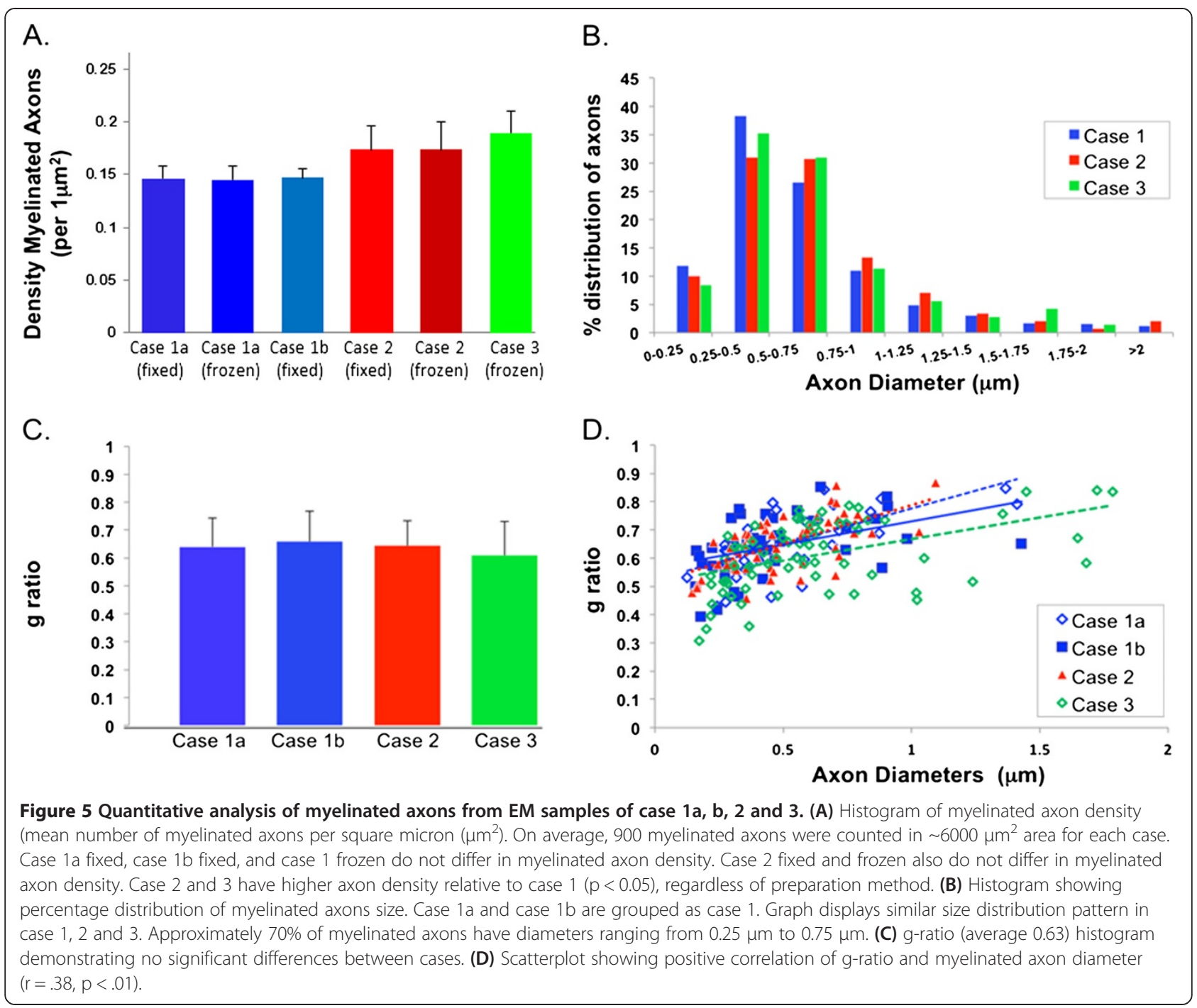

size range. The axon size range also confirmed the previous human frontal white matter study carried out by Zikopoulos and Barbas [5] with average axonal diameter of approximately $0.80-0.9 \mu \mathrm{m}$. These measures are also consistent with previous findings in other species; for example in fornix $(0.81 \mu \mathrm{m})$, pyramid tract $(0.9 \mu \mathrm{m})$ and optic tract $(0.88 \mu \mathrm{m})$ of the guinea pig [18]. The thickness of the myelin sheath (as measured by g-ratio) also does not differ between the six samples. Surprisingly, our quantitative data also suggest that larger axons may have thinner myelin sheaths, however further study on a larger sample of cases would be required to confirm this finding.

From an evolutionary perspective, thicker myelinated axons may imply that longer connections and more reliable axonal conductions contribute significantly to the advancement of cognitive functions in higher mammals, including human. Interestingly, human brain g-ratio numbers differ from rodent brains [11], indicating that overall myelin sheath layers are thicker in human. The loss of myelinated axons during aging also reflects declining cognitive ability $[19,20]$. Moreover, loss of myelin layers in neurological diseases including multiple sclerosis and autism [5,21] strongly implicates that structural integrity of myelinated axons are crucial for maintaining normal neuronal function and more detailed analysis of normal and diseased brain tissues are highly required for future investigations.

\section{Applications for the study of human brain pathology}

The need for EM analyses of human brain white matter has increased recently due to theories that many neurodevelopmental and psychiatric disorders have disruptions in neuronal connectivity. For example, DTI studies of schizophrenia have reported increased radial diffusivity that may reflect deficits in myelin integrity [22]. However, currently the low spatial resolution of DTI presents a considerable challenge of dissociating crossed fibers within a single voxel, and therefore limiting the detection of changes in axonal or myelin structure. 
A number of schizophrenia studies suggest abnormalities in the ultrastructure of myelin sheaths, reductions in oligodendrocyte numbers in some but not all brain regions, and dysregulation of myelin-associated gene expression, which could have profound effects on neuronal signaling [23-27]. One EM study found alterations in the ultrastructure of myelinated fibers and oligodendrocytes in prefrontal cortex [6]; however more studies are necessary in conjunction with immunohistochemistry [28] to characterize deficits in neuronal connectivity in patients with schizophrenia.

In autism spectrum disorder, numerous DTI studies support the theory of aberrant development of brain connectivity due to altered axonal microstructure [29]. In the only EM study of the autism brain published to date, Zikopoulos and Barbas [5] found evidence to support the hypothesis that myelinated axons of neurons in the prefrontal cortex may have increased local connectivity and decreased long-range connectivity. Specifically, they found significantly fewer large axons in the deep white matter below anterior cingulate cortex and a significantly greater density of smaller axons in superficial white matter of the same region. Unfortunately due to the lack of quality fixed tissue blocks available, this study was limited to a small sample size of five autism brains (one case also diagnosed with schizophrenia) and four control brains. Zikopoulos and Barbas [5], in addition to a large number of DTI studies [30], clearly demonstrate the need for larger scale studies of white matter ultrastructural pathology in multiple brain regions, given that ASD neuropathology is not limited to the prefrontal cortex [31]. From a technique perspective, the Zikopoulos and Barbas [5] study and the current study's methods differed; in that they postfixed samples with microwave to enhance immunolabeling. In comparison to their approach, we preserved brain tissues in paraformaldehyde plus glutaraldehyde solution to omit microwaving procedures. In addition, their study was limited to fixed samples whereas the current study also demonstrated this method on frozen sections. With the method presented here, white matter ultrastructure was also well preserved, could be quantified, and combined with immunohistochemical and stereological studies within the same brain section. Given that high quality autism brain tissue, and in particular pediatric tissue, is likely more difficult to acquire than any other neurological or psychiatric disorder, novel methods such as the one presented here are necessary to shed light on the underlying neuropathological basis of aberrant brain connectivity in this disorder.

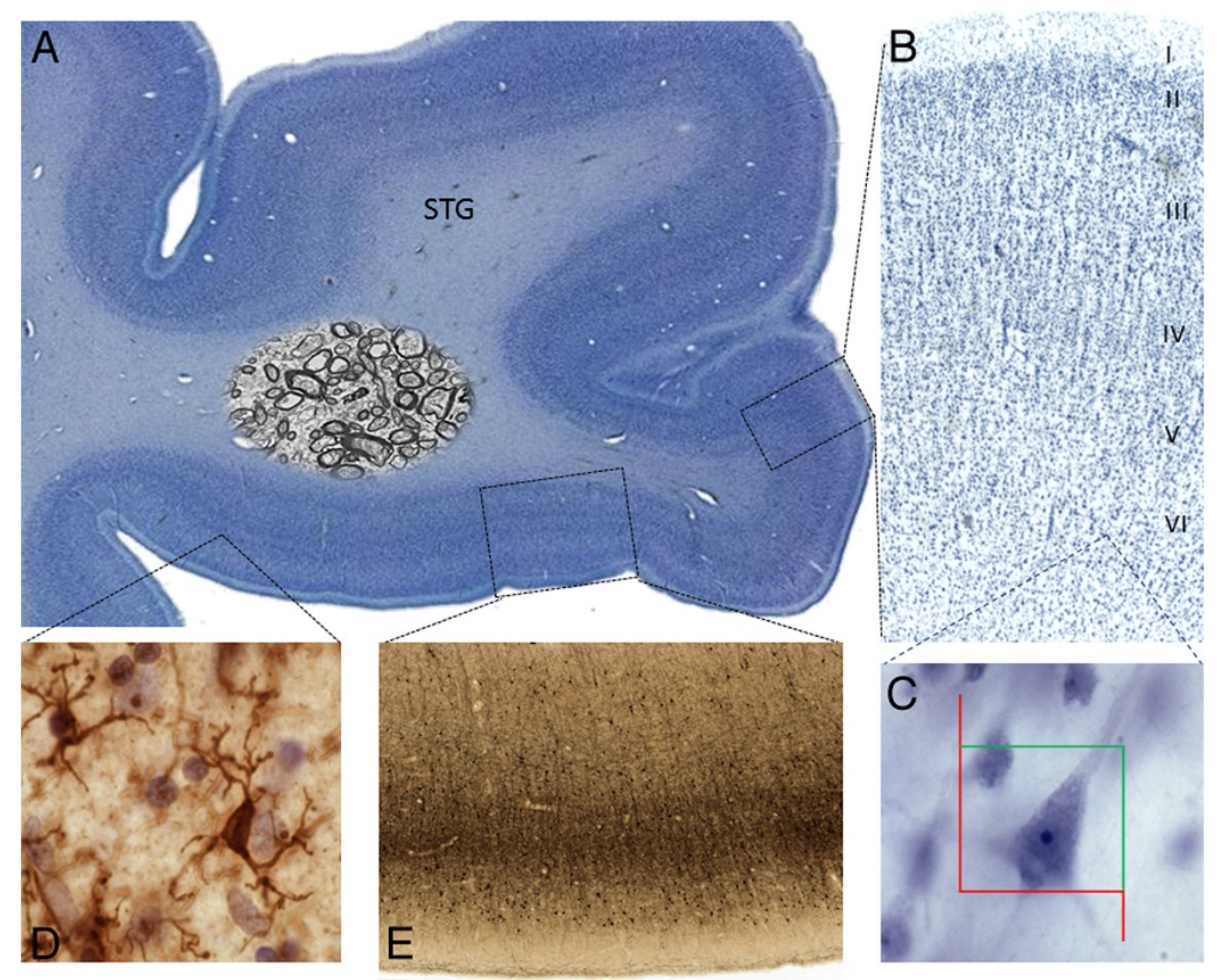

Figure 6 Examples of potential preparations that can be applied to fixed-frozen sections adjacent to the STG section used for EM on case 3. (A) Nissl (thionin) cell body stain. (B) Higher magnification of (A) showing layers of STG cortex. (C) Example of pyramidal neuron from (B) at 100x magnification used for stereological estimation of neuron number. (D) Iba-1 stained section for microglia. (E) Parvalbumin stained section through STG of case 3. Additional protocols available upon request. 


\section{Conclusions}

The method presented here for preparing human brain long term fixed and fixed-frozen cut sections for EM opens an enormous opportunity for future large scale quantitative studies of axonal ultrastructure. Significantly, we can combine light microscopy for immunocytochemical or other histological labeling/stereological studies using the same serial sections (Figure 6 of case 3); thus a correlative neuropathological characterization can be carried out at the cellular and subcellular level. In addition, we can now use the same technique on human postmortem brains and non-human primate animal models of human disorders, such as autism and schizophrenia, to assess common features and the validity of the model.

A critical question is: Can measures from varying preparations be combined? In general, the preservation of white matter ultrastructure was consistent within and across cases. There was an approximately $15 \%$ reduction in axon density in case 1 relative to cases 2 and 3, however this finding appeared to be irrespective of freezing. Although the extra-axonal space varied from case to case, the axonal ultrastructure and axon size distribution were not compromised and are also comparable to results from previous studies on human brain samples [5,32]. Moreover, a similar g-ratio number indicates that myelin sheath thickness and integrity are not significantly altered due to long term fixation or sectioning method used. Therefore, although it is possible for measures of axon size distribution to be combined across methods, ideally, brain samples should be prepared with as consistent a protocol as possible. Taken together, this study demonstrates that long term formalin fixed and frozen cut brain sections can be used for EM study of ultrastructural analyses, however caution should be taken in interpreting the results of axon density and considerations made for other possible confounds when combining various preparation methods.

\section{Competing interest}

The authors declare that the research was conducted in the absence of any commercial or financial relationships that could be construed as a potential conflict of interest.

\section{Author contributions}

$X L$ made a substantial contribution to the design of the study, acquisition and analysis of data, and drafted and revised the manuscript. CMS made a substantial contribution to the conception and design of the study, interpretation of data, and revised the manuscript. Both authors gave final approval of the version to be published and agreed to be accountable for all aspects of the work.

\section{Acknowledgements}

The authors thank Kenneth Lam for his assistance in data collection, Dr. Nicole Barger for her assistance in tissue acquisition, Dr. David Amaral for his consultation on the use of frozen tissue with EM methods, and Aaron Lee and Alicja Omanska for their assistance in figure preparation. Tissue samples were provided by Dr. Claudia Greco, UC Davis School of Medicine Department of Pathology. The authors gratefully acknowledge the support of the UC Davis MIND Institute, UC Davis Center for Neuroscience, and the National Institute of Mental Health MH097236 (Schumann). We are deeply indebted to the families of our donors who have made this study possible.

\section{Author details}

${ }^{1}$ University of California, Davis, School of Medicine, Department of Pathology and Laboratory Medicine; Electron Microscopy Laboratory, Sacramento, USA. ${ }^{2}$ University of California, Davis, School of Medicine, Department of Psychiatry and Behavioral Sciences, MIND Institute, Sacramento, USA. ${ }^{3}$ UC Davis MIND Institute, 2805 20th Street, Sacramento, CA 95817, USA.

Received: 6 February 2014 Accepted: 26 March 2014

Published: 11 April 2014

\section{References}

1. Fields RD: White matter in learning, cognition and psychiatric disorders. Trends Neurosci 2008, 31:361-370.

2. LaMantia AS, Rakic P: Axon overproduction and elimination in the corpus callosum of the developing rhesus monkey. J Neurosci 1990, 10:2156-2175.

3. Makinodan M, Rosen KM, Ito S, Corfas G: A critical period for social experience-dependent oligodendrocyte maturation and myelination. Science 2012, 337:1357-1360.

4. Nave KA: Neuroscience: An ageing view of myelin repair. Nature 2008, 455:478-479.

5. Zikopoulos B, Barbas $\mathrm{H}$ : Changes in prefrontal axons may disrupt the network in autism. J Neurosci 2010, 30:14595-14609.

6. Uranova NA, Vikhreva OV, Rachmanova VI, Orlovskaya DD: Ultrastructural alterations of myelinated fibers and oligodendrocytes in the prefrontal cortex in schizophrenia: a postmortem morphometric study. Schizophr Res Treatment 2011, 2011:325789.

7. Concha L, Livy DJ, Beaulieu C, Wheatley BM, Gross DW: In vivo diffusion tensor imaging and histopathology of the fimbria-fornix in temporal lobe epilepsy. J Neurosci 2010, 30:996-1002

8. Freese $J \mathrm{~L}$, Amaral DG: The organization of projections from the amygdala to visual cortical areas TE and V1 in the macaque monkey. J Comp Neurol 2005, 486:295-317.

9. Schumann CM, Amaral DG: Stereological estimation of the number of neurons in the human amygdaloid complex. J Comp Neurol 2005, 491:320-329.

10. Peters A, Palay S, Webster H: The Fine Structure of the Nervous System. New York: Oxford University Press; 1991

11. Chomiak T, Hu B: What is the optimal value of the g-ratio for myelinated fibers in the rat CNS? A theoretical approach. PLoS One 2009, 4:e7754.

12. Marcus J, Honigbaum S, Shroff S, Honke K, Rosenbluth J, Dupree JL: Sulfatide is essential for the maintenance of CNS myelin and axon structure. Glia 2006, 53:372-381.

13. Freese $J$, Amaral DG: Synaptic organization of projections from the amygdala to visual cortical areas TE and V1 in the macaque monkey. J Comp Neurol 2006, 496:655-667.

14. Liu XB, Honda CN, Jones EG: Distribution of four types of synapse on physiologically identified relay neurons in the ventral posterior thalamic nucleus of the cat. J Comp Neurol 1995, 352:69-91.

15. Liu XB, Warren RA, Jones EG: Synaptic distribution of afferents from reticular nucleus in ventroposterior nucleus of cat thalamus. J Comp Neurol 1995, 352:187-202.

16. Needleman LA, Liu XB, El-Sabeawy F, Jones EG, McAllister AK: MHC class I molecules are present both pre- and postsynaptically in the visual cortex during postnatal development and in adulthood. Proc Natl Acad Sci U S A 2010, 107:16999-17004.

17. Shen Y, Liu XB, Pleasure DE, Deng W: Axon-glia synapses are highly vulnerable to white matter injury in the developing brain. J Neurosci Res 2012, 90:105-121.

18. Perge JA, Niven JE, Mugnaini E, Balasubramanian V, Sterling P: Why do axons differ in caliber? J Neurosci 2012, 32:626-638.

19. Marner L, Nyengaard JR, Tang Y, Pakkenberg B: Marked loss of myelinated nerve fibers in the human brain with age. J Comp Neurol 2003, 462:144-152.

20. Zhang K, Sejnowski TJ: A universal scaling law between gray matter and white matter of cerebal cortex. Proc Natl Acad Sci USA 2000, 97:5621-5626.

21. Nave KA: Myelination and support of axonal integrity by glia. Nature 2010, 468:244-252

22. Alba-Ferrara LM, de Erausquin GA: What does anisotropy measure? Insights from increased and decreased anisotropy in selective fiber tracts in schizophrenia. Front Integr Neurosci 2013, 7:9.

23. Fujino J, Takahashi H, Miyata J, Sugihara G, Kubota M, Sasamoto A, Fujiwara $H$, Aso T, Fukuyama $H$, Murai T: Impaired empathic abilities and reduced 
white matter integrity in schizophrenia. Prog Neuropsychopharmacol Biol Psychiatry 2014, 48:117-123.

24. Hoistad M, Heinsen H, Wicinski B, Schmitz C, Hof PR: Stereological assessment of the dorsal anterior cingulate cortex in schizophrenia: absence of changes in neuronal and glial densities. Neuropathol Appl Neurobiol 2013, 39:348-361.

25. Lewis DA: Cortical circuit dysfunction and cognitive deficits in schizophrenia-implications for preemptive interventions. Eur J Neurosci 2012, 35:1871-1878.

26. Takahashi N, Sakurai T, Davis KL, Buxbaum JD: Linking oligodendrocyte and myelin dysfunction to neurocircuitry abnormalities in schizophrenia. Prog Neurobiol 2011, 93:13-24.

27. Voineskos AN, Foussias G, Lerch J, Felsky D, Remington G, Rajji TK, Lobaugh $\mathrm{N}$, Pollock BG, Mulsant BH: Neuroimaging evidence for the deficit subtype of schizophrenia. JAMA Psychiatry 2013, 70:472-480.

28. Glausier JR, Fish KN, Lewis DA: Altered parvalbumin basket cell inputs in the dorsolateral prefrontal cortex of schizophrenia subjects. $\mathrm{Mol}$ Psychiatry 2014, 19:140

29. McFadden K, Minshew NJ: Evidence for dysregulation of axonal growth and guidance in the etiology of ASD. Front Hum Neurosci 2013, 7:671.

30. Zikopoulos B, Barbas $\mathrm{H}$ : Altered neural connectivity in excitatory and inhibitory cortical circuits in autism. Front Hum Neurosci 2013, 7:609.

31. Schumann CM, Nordahl CW: Bridging the gap between MRI and postmortem research in autism. Brain Res 2011, 1380:175-186.

32. Pakkenberg B, Pelvig D, Marner L, Bundgaard MJ, Gundersen HJ, Nyengaard $J R$, Regeur L: Aging and the human neocortex. Exp Gerontol 2003, 38:95-99.

doi:10.1186/2051-5960-2-42

Cite this article as: Liu and Schumann: Optimization of electron microscopy for human brains with long-term fixation and fixed-frozen sections. Acta Neuropathologica Communications 2014 2:42.

\section{Submit your next manuscript to BioMed Central and take full advantage of:}

- Convenient online submission

- Thorough peer review

- No space constraints or color figure charges

- Immediate publication on acceptance

- Inclusion in PubMed, CAS, Scopus and Google Scholar

- Research which is freely available for redistribution 